\title{
Remediación de Aguas Contaminadas, Utilizando Dióxido de Titanio Modificado con Hierro como Fotocatalizador.
}

\section{Remediation of Contaminated Water, Using Iron Doped Titanium Dioxide as a Photocatalyst.}

\section{Pablo A. Ochoa Rodríguez}

Centro de Investigación y Tecnología Química (CITeQ) - UTN CONICET, Maestro López esq. Cruz Roja s/n, Córdoba Capital - Argentina

pochoa@frc.utn.edu.ar

\section{Gina Pecchi}

Departamento de Fisicoquímica - Facultad de Ciencias Químicas - Universidad de Concepción PostOffice 160-C - Concepción - Chile

ginapecchi@gmail.com

\section{Sandra G. Casuscelli}

Centro de Investigación y Tecnología Química (CITeQ) - UTN CONICET, Maestro López esq. Cruz Roja s/n, Córdoba Capital - Argentina

scasuscelli@frc.utn.edu.ar

\section{Verónica R. Elías}

Centro de Investigación y Tecnología Química (CITeQ) - UTN CONICET, Maestro López esq. Cruz Roja s/n, Córdoba Capital - Argentina

velias@frc.utn.edu.ar

\section{Griselda A. Eimer}

Centro de Investigación y Tecnología Química (CITeQ) - UTN CONICET, Maestro López esq. Cruz Roja s/n, Córdoba Capital - Argentina

geimer@frc.utn.edu.ar 


\title{
Resumen
}

Materiales de dióxido de titanio (TiO2) fueron empleados como catalizadores en la degradación acuosa fotocatalítica del ácido naranja 7 (AO7). Los materiales fueron sintetizados empleando la técnica sol-gel y bajo condiciones hidrotérmicas. Con el objetivo de lograr un uso más eficiente de la luz solar, los materiales fueron modificados con distintas cargas de hierro. Los estudios de caracterización dieron cuenta de la presencia de la fase anatasa en la estructura cristalina interna, de la naturaleza mesoporosa, y del desplazamiento de la absorción hacia mayores longitudes de onda con el agregado de hierro. Las reacciones fueron llevadas a cabo en un reactor batch irradiado desde sus laterales. El catalizador modificado con $0,1 \% \mathrm{P} / \mathrm{P}$ de hierro resultó ser el más activo, elevando el porcentaje de degradación del contaminante hasta $54 \%$, en relación al $26 \%$ obtenido con el TiO2 sin modificar. A contenidos superiores de metal, la actividad disminuyó aún por debajo del $26 \%$.

Palabras Claves: titania; fotocatálisis; contaminantes orgánicos; degradación

\begin{abstract}
Titanium dioxide (TiO2) materials were used as catalysts in the photocatalytic aqueous degradation of acid orange 7 (AO7). The materials were synthesized using the sol-gel technique and under hydrothermal conditions. In order to achieve a more efficient use of sunlight, the materials were modified with different loads of iron. The characterization studies showed the presence of the anatase phase in the internal crystalline structure, the materials' mesoporous nature, and the displacement to longer wavelengths with the addition of iron. The reactions were carried out in a batch reactor irradiated from its sides. The catalyst modified with an iron load of $0.1 \%$ wt. was found to be the most active, increasing the percentage of the contaminant degradation to $54 \%$, in relation to the $26 \%$ obtained with the unmodified TiO2. At higher metal contents, the activity decreased even below $26 \%$.
\end{abstract}

Keywords: titanium; photocatalysis; organic pollutants; degradation

\section{Introducción y Objetivos}

Recientemente, ha adquirido gran importancia el estudio de técnicas para la remediación de aguas subterráneas contaminadas con materia orgánica refractaria y persistente. Una de las alternativas propuestas, como complemento a los tratamientos primarios y secundarios, incluye a los Procesos Avanzados de Oxidación. El fundamento de la técnica es lograr generar, en el medio acuoso de reacción, especies radicalarias (de elevado poder oxidante) que ataquen a los contaminantes promoviendo su degradación (disminución de la concentración del contaminante en el medio) y mineralización (conversión de la materia orgánica a dióxido de carbono y agua). Para esto, es necesario utilizar un sólido semiconductor como catalizador que, al ser excitado con energía radiante de determinada frecuencia, promueva un electrón desde su capa de valencia a la capa de conducción, dejando un hueco positivo. La interacción de estas especies positivas y negativas con el oxígeno y agua del medio, da lugar a la formación del radical hidroxilo $\left(\mathrm{OH}^{*}\right)$, especie que finalmente atacará al contaminante 
(Hoffmann, M.,1995). El éxito de la técnica radica en impedir o retardar el proceso de recombinación del par hueco/electrón, para poder garantizar la presencia de los radicales. Un semiconductor ampliamente utilizado es el TiO2 (dióxido de titanio o titania). El mismo puede ser activado cuando absorbe radiación de longitudes de onda $(\lambda)<$ menores a $400 \mathrm{~nm}$, es decir del rango ultravioleta del espectro, ya que su band gap es de 3,2 eV (Fujishima, A., 2000). Para poder hacer un mejor uso y aprovechamiento de la luz solar, se dopa la titania con distintos elementos metálicos y no metálicos. Entre ellos, se encuentran el C, N, Fe y $\mathrm{Cu}$. El propósito es lograr que disminuya el band gap, para conseguir que el TiO2 absorba energía en el rango visible, de $\lambda>400 \mathrm{~nm}$ (Choi, H., 2007).

En este contexto, en el siguiente trabajo se propone la síntesis de dióxido de titanio de elevada área específica, para utilizar en reacciones de degradación del contaminante AO7 (ácido naranja 7), presente en efluentes vertidos a cursos de agua por la industria textil. Se siguió el método sol-gel, y se trabajó a una temperatura de $85^{\circ} \mathrm{C}$ con calcinación final. Para incorporar el hierro en la estructura se utilizó el método de impregnación húmeda.

Los catalizadores fueron caracterizados mediante las técnicas de difracción de rayos $\mathrm{X}$ (DRX), espectrometría UV-Vis, adsorción-desorción con N2, y microscopía electrónica de barrido (SEM).

Las reacciones fueron llevadas a cabo en un reactor batch, y se trabajó sólo con radiación visible. Se evaluó el efecto de la carga de hierro en la actividad del fotocatalizador, evaluando la degradación alcanzada al cabo de cinco horas de ensayo.

\section{Metodología}

\section{Síntesis y modificación de los catalizadores}

Como fuente de titanio se utilizó n-butóxido de titanio $(\mathrm{Ti}(\mathrm{OBu}) 4)$ y como agente director de estructura Pluronic P123. En una primera instancia se prepara la solución S1 disolviendo el P123 en etanol. Una segunda solución S2 se lleva a cabo disolviendo la fuente de titanio en ácido acético. Al cabo de cuatro horas de agitación a temperatura ambiente, se añade gota a gota S1 en S2, para dejar después agitando por 24 h. A continuación, el sistema es llevado a autoclave en un reactor de teflón bajo presión autogenerada a $85^{\circ} \mathrm{C}$. Luego de $48 \mathrm{~h}$ de tratamiento, se recupera el sólido obtenido mediante filtración para posterior calcinación a $450^{\circ} \mathrm{C}$ durante $4 \mathrm{~h}$ y en atmósfera de aire. El sólido obtenido (matriz) se denomina MT.

Para incorporar el heteroátomo de hierro en la estructura de titania, se utilizó el método de impregnación húmeda utilizando un evaporador rotatorio. Para esto, se coloca en un balón cantidades adecuadas de matriz y solución de $\mathrm{FeCl} 3{ }^{*} 6 \mathrm{H} 2 \mathrm{O}$ de concentración acorde a la cantidad de hierro que se desea impregnar. Nuevamente, los materiales se llevan a calcinación bajo aire a $450^{\circ} \mathrm{C}$. Los catalizadores se denominan: Fe/MT(x), donde en $\mathrm{x}$ se indica la carga teórica de hierro $(0,0125 ; 0,025 ; 0,1$ o $1 \% \mathrm{P} / \mathrm{P})$.

\section{Estudios de caracterización}

Los sólidos fueron caracterizados mediante difracción de Rayos X (DRX) a alto ángulo (de 20 a $80^{\circ}$ ), en un equipo Philips PW1800/10 con monocromador de grafito y tubo con ánodo de cobre. El barrido espectral de las muestras fue llevado a cabo en un espectrofotómetro Jasco V-650 con esfera integradora. El valor del área específica, diámetro de poros, y tipo de isotermas, fue obtenido a partir del estudio de fisisorción con N2 (con los métodos de 
BET para el área y BJH para el diámetro de poros), realizado en un equipo Tristar II 3020 de Micromeritics. La morfología de los materiales fue observada por microscopía electrónica de barrido (SEM) en un equipo JEOL JSM-6380 LV (20 kV).

\section{Reacciones de degradación}

Para llevar a cabo las reacciones de degradación del AO7, se utilizó un reactor batch descripto en (Elías, V.,2011). El mismo es irradiado, desde sus laterales, por cuatro lámparas UV-Vis. Al trabajar con radiación visible, en el espacio que hay entre el reactor de vidrio y las lámparas, se colocan filtros de acrílico que impiden el paso de parte de la radiación UV. Se partió de una concentración inicial de contaminante (C0) de 20 ppm, y 1 g/L de catalizador. A regulares intervalos de tiempo, se fue midiendo el valor de la concentración de AO7 (C) a través de la medición de la absorbancia del medio de reacción a $485 \mathrm{~nm}$, en el equipo Jasco V-650. Para conocer el nivel de mineralización, se determinó el contenido de Carbono Orgánico Total (TOC) al comienzo y al final de la reacción fotocatalítica, de cinco horas de duración. Esto se llevó a cabo en un equipo TOC 5050A Shimadzu.

\section{Resultados y Discusión}

\section{Difracción de rayos $\mathrm{X}$}

Los patrones de difracción de rayos X demuestran la existencia, al 100\%, de la fase anatasa para todos los catalizadores. Para las muestras con hierro, no se observaron picos adicionales correspondientes a la formación de óxidos de hierro.

La fase anatasa es la activa fotocatalíticamente, y es asociada a los picos que aparecen a $25,3^{\circ} ; 37,8^{\circ} ; 48^{\circ} ; 53,8^{\circ} ; 54,9^{\circ}$ y $62,7^{\circ}$. No se registran picos correspondientes a la fase rutilo (Wu, Y.,2010). 


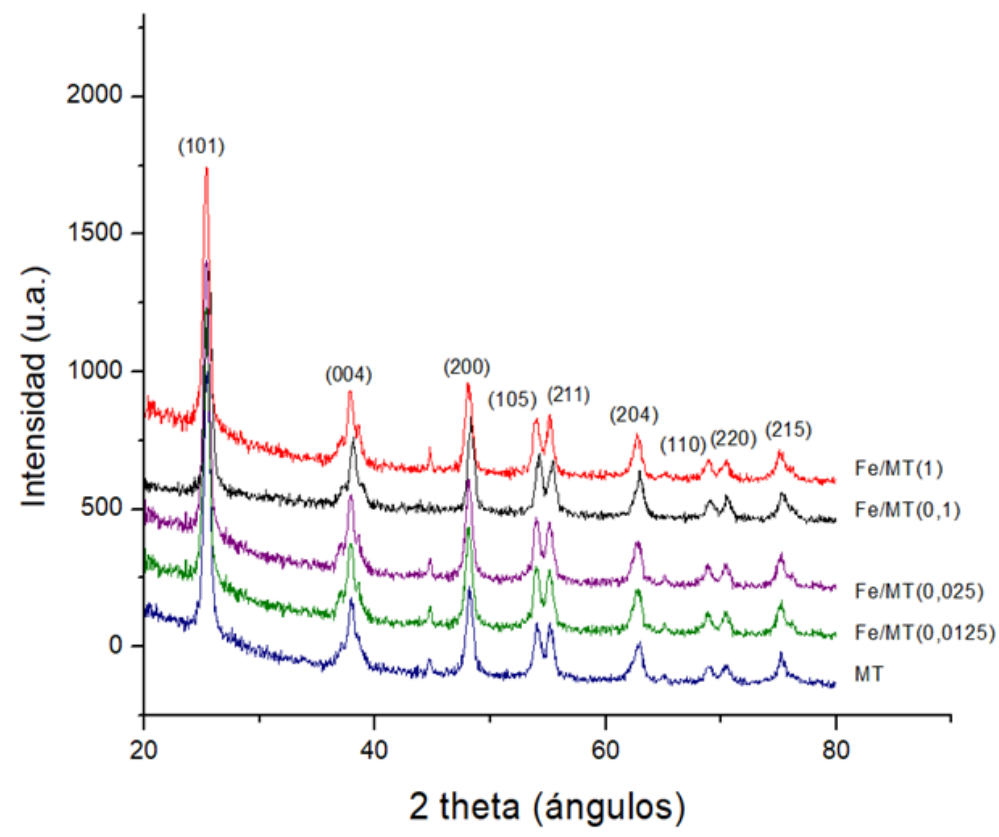

Figura 1. Patrones de difracción de rayos $\mathrm{X}$ de los sólidos.

\section{Espectrofotometría UV-Vis}

En cuanto al barrido espectral, se aprecia que el catalizador sin hierro no absorbe radiación a longitudes de onda superiores a $400 \mathrm{~nm}$. A medida que la carga de hierro aumenta, es mayor el desplazamiento hacia longitudes superiores. Es decir, el agregado de hierro posibilita la absorción de los sólidos en el rango visible del espectro. Esto es debido a la introducción de estados electrónicos entre especies catiónicas de hierro, que se extienden a lo largo de la banda prohibida de la titania pura (Moradi, H., 2016). 


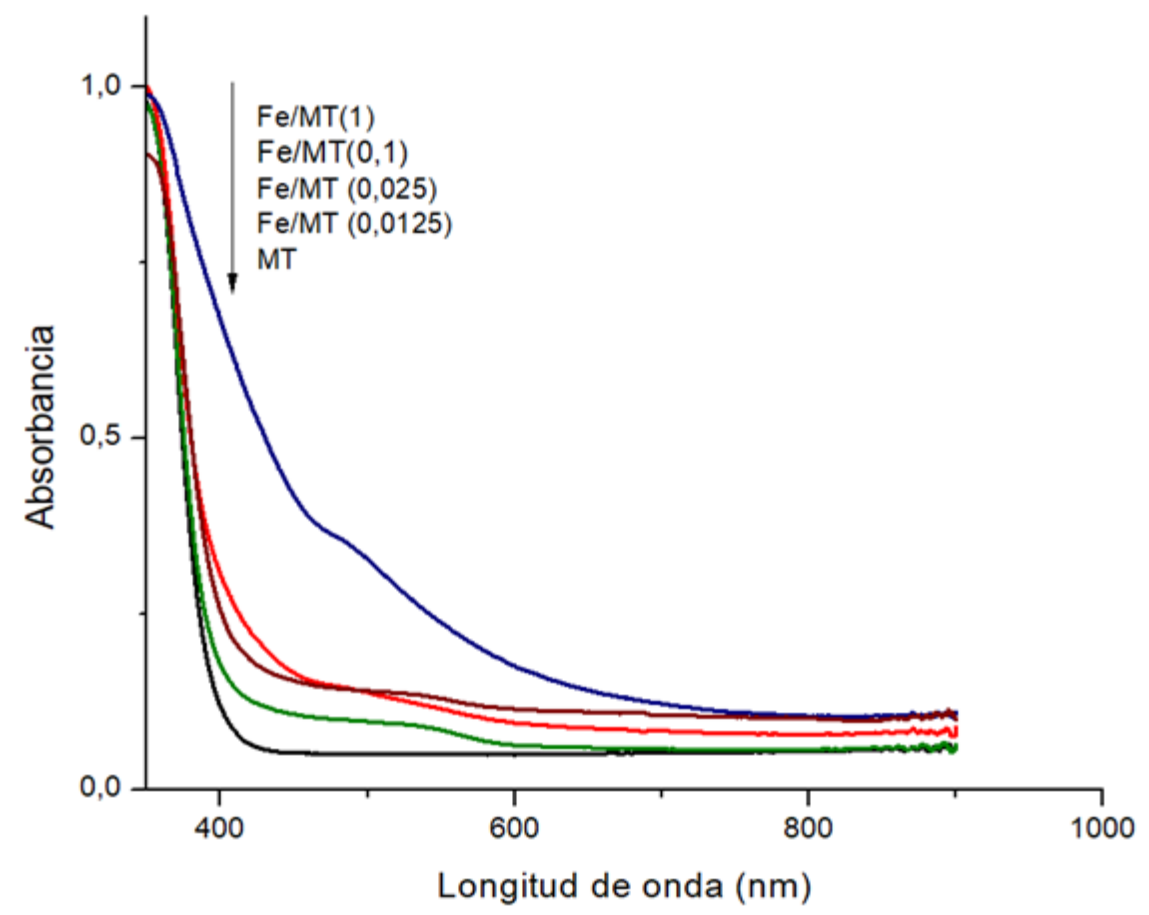

Figura 2. Espectros de absorción de los catalizadores.

\section{Adsorción-desorción con nitrógeno. Área y tamaño de poro.}

Del estudio de caracterización de fisisorción con nitrógeno, se deduce que las isotermas obtenidas para todas las muestras corresponden a isotermas tipo IV, las cuales son características de materiales mesoporosos (Jung Li, C., 2013). En cuanto al tamaño de esos poros, y el área específica de cada sólido, se presentan los valores en la siguiente tabla.

\begin{tabular}{|c|c|c|}
\hline Catalizador & $\begin{array}{c}\text { Área específica } \\
{\left[\mathrm{m}^{2} / \mathrm{g}\right]}\end{array}$ & $\begin{array}{c}\text { Tamaño de } \\
\text { poro }[\mathrm{nm}]\end{array}$ \\
\hline $\mathrm{MT}$ & 87 & 8 \\
\hline Fe/MT $(0,0125)$ & 60 & 8 \\
\hline Fe/MT $(0,025)$ & 60 & 8 \\
\hline Fe/MT $(0,1)$ & 52 & 6 \\
\hline Fe/MT $(1)$ & 47 & 4 \\
\hline
\end{tabular}

Tabla 1. Características morfológicas de las muestras 


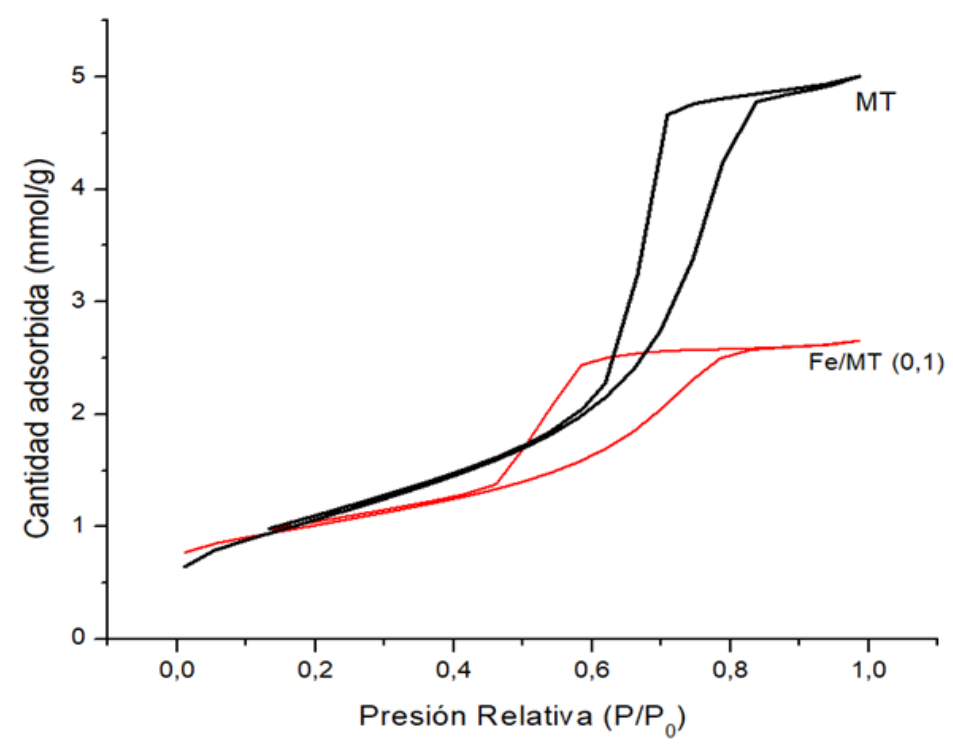

Figura 3. Isotermas de adsorción-desorción de la matriz, y del sólido más activo.

\section{Microscopía electrónica de barrido (SEM)}

Las imágenes muestran partículas esféricas, de textura suave y libres de agrietado. El añadido de hierro, no parece modificar esta consistencia.

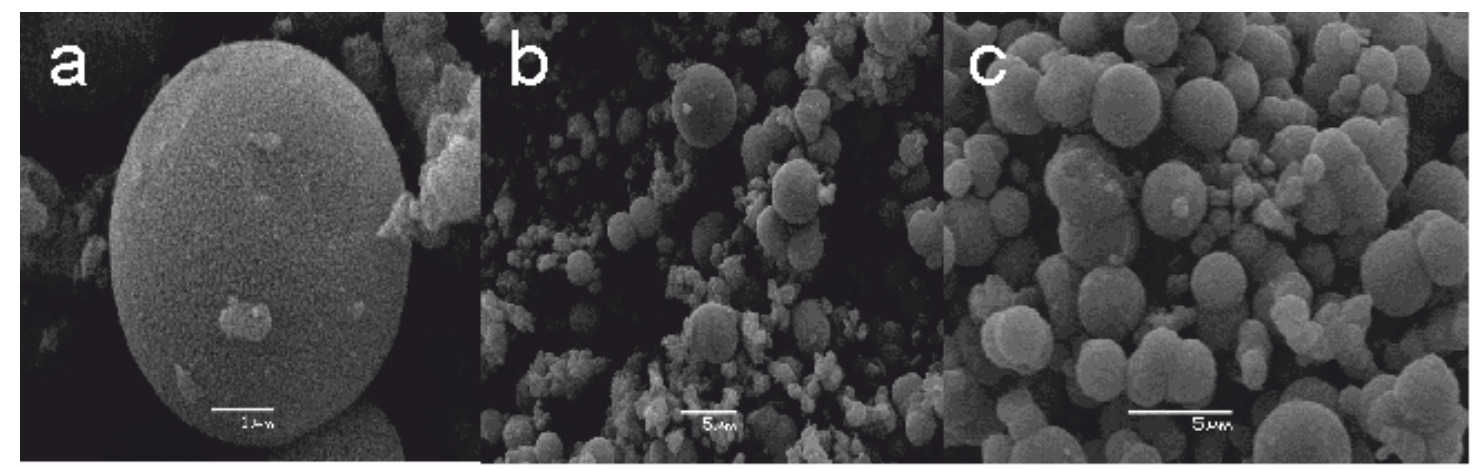

Figura 4. Imágenes SEM de a) b) MT c) Fe/MT $(0,1)$

\section{Actividad fotocatalítica}

Bajo radiación visible, con el catalizador MT sólo se consigue un $26 \%$ de degradación. A medida que se incrementa la carga de hierro impregnada en la estructura del sólido, mejora la actividad. Esto es atribuido a la mayor absorción que existe en el rango visible, debido 
a la presencia de este metal, que da lugar a la existencia de transiciones electrónicas que causan la disminución del band gap con respecto al del TiO2 puro. Es importante notar que al aumentar la concentración del hierro desde un 0,0125 a 0,025 y hasta un $0,1 \% \mathrm{P} / \mathrm{P}$ se observó un aumento en la actividad del material, porque fue aumentando el número de sitios metálicos que dan lugar a la absorción más eficiente de la radiación. A su vez, al no ser elevadas las cantidades de hierro incorporadas (menores a $0,1 \% \mathrm{P} / \mathrm{P}$ ), no se formaron especies de óxidos de hierro de mayor tamaño que pudieran actuar como centros de recombinación de los pares huecos positivos/electrones (Sood, S., 2015). El material que presentó la mayor actividad, bajo luz visible, fue el Fe/MT (0,1), consiguiéndose una degradación del orden del $54 \%$. En cuanto a la mineralización, para todos los casos se alcanzaron porcentajes del 10\%, por lo que es necesario continuar optimizando el material de manera que se dé lugar a un proceso que alcance la eliminación total del contaminante del medio. Cargas elevadas (1\%) de heteroátomo en la matriz pura, produce un detrimento en la actividad fotocatalítica, aún por debajo del $26 \%$.

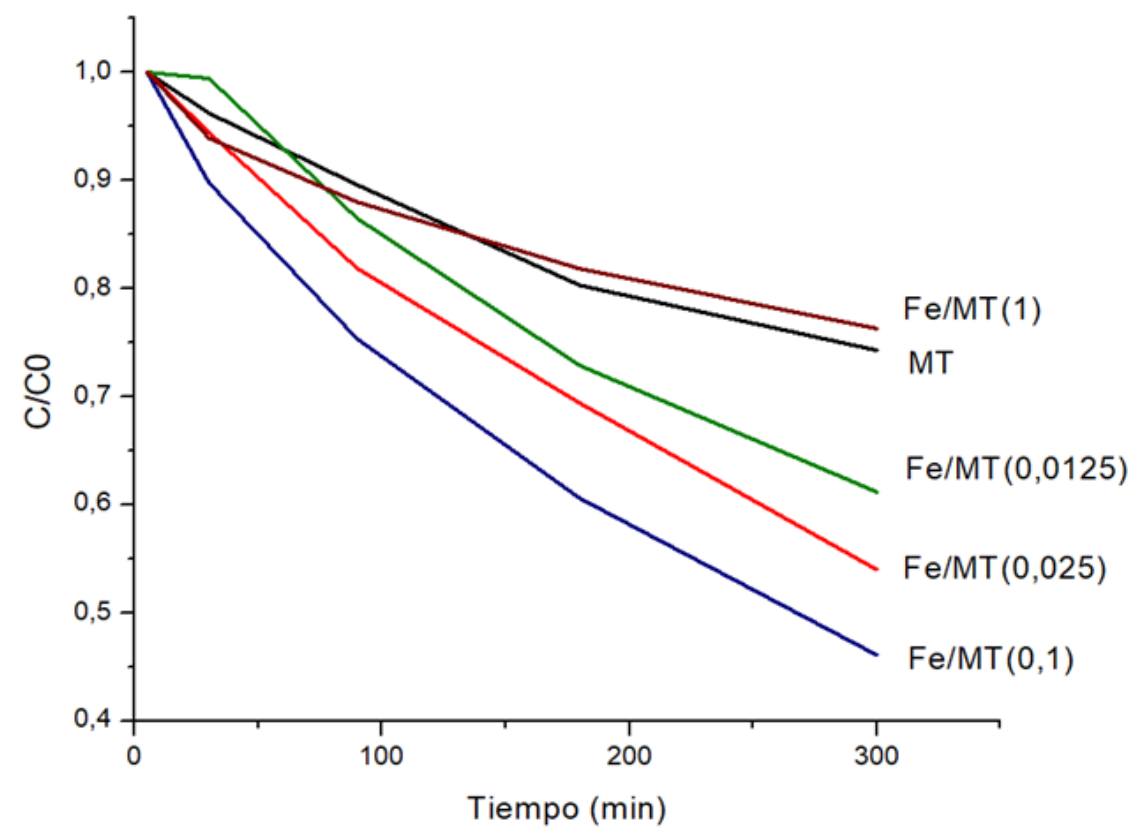

Figura 5. Actividad fotocatalítica de los materiales ensayados, bajo luz visible

\section{Conclusiones}

Lograron sintetizarse materiales nano-estructurados de TiO2, para luego modificarlos con hierro, y evaluar su actividad catalítica en Procesos Avanzados de Oxidación. Se utilizó la técnica sol-gel para la síntesis, y el método de impregnación húmeda para la incorporación del heteroátomo. Los estudios de caracterización dieron cuenta de la existencia de la 
fase anatasa y la mesoporosidad en todos los catalizadores. El estudio de espectrofotometría permitió aseverar que el desplazamiento hacia longitudes de onda mayores es posible gracias al agregado de hierro en la estructura de la titania. Luego de llevar a cabo los ensayos de degradación fotocatalítica del contaminante AO7, bajo luz visible, es posible inferir que la incorporación de hierro a bajas concentraciones mejora la actividad de la matriz. En este sentido, el catalizador Fe/MT $(0,1)$ logró incrementar el nivel de degradación hasta un 54\%, en relación al 26\% alcanzado con MT. Este fenómeno fue asociado a la mayor absorción mostrada por el material en el rango visible del espectro, y a la no formación de óxidos que actúan como centros de recombinación de las especies. Estos óxidos se detectan cuando son elevadas las concentraciones de hierro impregnadas.

De este modo, sintetizar dióxido de titanio mesoporoso y modificarlo con bajas concentraciones de hierro empleando un método rápido (como lo es la impregnación húmeda), para luego utilizarlo en procesos de remediación ambiental con luz de baja energía, constituye un avance para la puesta en marcha de sistemas eco-compatibles. 


\section{Agradecimientos}

Al Centro de Investigación y Tecnología Química, Unidad Ejecutora de doble dependencia UTN-CONICET, por ser el principal lugar de trabajo experimental y académico.

\section{Referencias}

Hoffmann, M. (1995). "Environmental applications of semiconductor photocatalysis", Chemical Reviews, 95, 69-96.

Fujishima, A. (2000). "Titanium dioxide photocatalysis", Journal of Photochemistry and Photobiology A: Chemistry, C1, 1-21.

Choi, H. (2007). "Mesoporous nitrogen-doped TiO2 for the photocatalytic destruction of the cyanobacterial toxin microcystin-LR under visible light irradiation", Environmental Science \& Technology, 41, 7530-7535.

Elías, V. (2011). "Synthesis and Photocatalytic Activity of Titania-Loaded Transition MetalModified MCM-41 Molecular Sieves”, Topics in Catalysis, 54, 277-286.

$\mathrm{Wu}, \mathrm{Y}$. (2010). "Properties of carbon and iron modified TiO2 photocatalyst synthesized at low temperature and photodegradation of acid orange 7 under visible light”, Applied Surface Science, 256, 4260-4268.

Moradi, H. (2016). "Fabrication of Fe-doped TiO2 nanoparticles and investigation of photocatalytic decolorization of reactive red 198 under visible light irradiation", Ultrasonics Sonochemistry, 32, 314-319.

Jung Li, C. (2013). "Ordered Mesostructured Cu doped TiO2 spheres as active visible light driven photocatalysts for degration of Paracetamol”, Chemical Engineering Journal, http:// dx.doi.org/10.1016/j.cej.2013.10.027 (2013).

Sood, S. (2015). "Highly effective Fe-doped TiO2 nanoparticles photocatalysts for visible light driven photocatalytic degradation of toxic organic compounds", Journal of Colloid and Interface Science, 450, 213-223. 